\title{
Expression and cellular localization of cyclooxygenases and prostaglandin E synthases in the hemorrhagic brain
}

Tao $\mathrm{Wu}^{1,2}$, He $\mathrm{Wu}^{1,3}$, Jessica Wang ${ }^{1}$, Jian Wang ${ }^{1 *}$

\begin{abstract}
Background: Although cyclooxygenases (COX) and prostaglandin E synthases (PGES) have been implicated in ischemic stroke injury, little is known about their role in intracerebral hemorrhage (ICH)-induced brain damage. This study examines the expression and cellular localization of COX-1, COX-2, microsomal PGES-1 (mPGES-1), mPGES-2, and cytosolic PGES (CPGES) in mice that have undergone hemorrhagic brain injury.

Methods: ICH was induced in C57BL/6 mice by intrastriatal injection of collagenase. Expression and cellular localization of COX-1, COX-2, mPGES-1, mPGES-2, and CPGES were examined by immunofluorescence staining.

Results: In the hemorrhagic brain, COX-1, mPGES-2, and CPGES were expressed constitutively in neurons; COX-1 was also constitutively expressed in microglia. The immunoreactivity of COX-2 was increased in neurons and astrocytes surrounding blood vessels at $5 \mathrm{~h}$ and then tended to decrease in neurons and increase in astrocytes at 1 day. At 3 days after ICH, COX-2 was observed primarily in astrocytes but was absent in neurons. Interestingly, the immunoreactivity of mPGES-1 was increased in neurons in the ipsilateral cortex and astrocytes in the ipsilateral striatum at 1 day post-ICH; the immunoreactivity of astrocytic mPGES-1 further increased at 3 days.

Conclusion: Our data suggest that microglial COX-1, neuronal COX-2, and astrocytic COX-2 and mPGES-1 may work sequentially to affect ICH outcomes. These findings have implications for efforts to develop anti-inflammatory strategies that target COX/PGES pathways to reduce ICH-induced secondary brain damage.
\end{abstract}

\section{Background}

Intracerebral hemorrhage ( $\mathrm{ICH})$ is a subtype of stroke that carries high morbidity and mortality. ICH causes brain injury through primary physical disruption of adjacent tissue and the mass effect and secondary injury such as brain edema and inflammation [1,2]. Evidence from preclinical and clinical studies suggests that inflammatory mechanisms contribute to the progression of secondary brain injury after ICH [1,3]. Inflammatory cells that have the potential to promote hemorrhagic brain damage include blood-derived leukocytes and macrophages, resident microglia, astrocytes, and mast cells. Molecular components of the inflammatory response include prostaglandins, chemokines, cytokines,

\footnotetext{
* Correspondence: jwang79@jhmi.edu

'Department of Anesthesiology/Critical Care Medicine, Johns Hopkins

University, School of Medicine, Baltimore, MD, USA

Full list of author information is available at the end of the article
}

extracellular proteases, and reactive oxygen species [3-5].

Prostaglandin $\mathrm{E}_{2}\left(\mathrm{PGE}_{2}\right)$ is a key component in the initiation and propagation phases of the inflammatory process induced after various types of brain injury [6-8]. It is synthesized from arachidonic acid in two enzymatic steps. Cyclooxygenase (COX) catalyzes the conversion of arachidonic acid to $\mathrm{PGH}_{2}$, which is then converted to $\mathrm{PGE}_{2}$ by PGE synthase (PGES). COX is present in two isoforms, constitutive COX-1 and inducible COX-2. Under normal conditions, COX-1, which is expressed in numerous tissues, has been classically considered to be the isoform principally responsible for homeostatic prostaglandin synthesis [9]. In contrast, COX-2 is primarily expressed in glutamatergic neurons, wherein it is thought to participate in synaptic signaling [10]. Three major isozymes of PGES have been isolated: microsomal PGES (mPGES)-1, mPGES-2, and cytosolic PGES (cPGES). Of these, mPGES- 2 and cPGES are constitutively expressed
C Biomed Central

(c) 2011 Wu et al; licensee BioMed Central Ltd. This is an Open Access article distributed under the terms of the Creative Commons Attribution License (http://creativecommons.org/licenses/by/2.0), which permits unrestricted use, distribution, and reproduction in any medium, provided the original work is properly cited. 
in various cells and tissues; only mPGES- 1 can be induced by pro-inflammatory stimuli and in ischemic stroke models [7,11-13]. A recent study showed that COX-2 and mPGES- 1 are co-induced by excess glutamate in ischemic brain and that they act together to exacerbate stroke injury by amplifying $\mathrm{PGE}_{2}$ production [14].

Although $\mathrm{COX} /$ prostaglandin signaling has been implicated in the pathologic progression of ischemic stroke [15-17], research on their role in ICH is limited. To our knowledge, no reports have been published regarding expression of COX-1 and PGES isoforms in $\mathrm{ICH}$. Two studies have been published on COX-2 expression in blood models of $\mathrm{ICH}$ in rats, but the results are conflicting. One showed COX-2 immunoreactivity to be increased from $6 \mathrm{~h}$ to 3 days after $\mathrm{ICH}$ [18], whereas the second showed a transient increase in COX-2 from 1 to $3 \mathrm{~h}$ after $\mathrm{ICH}$ followed by a significant down-regulation [19]. To lay a foundation for understanding the roles of COX and PGES isoforms in ICH pathology, here we characterized the expression and cellular localization of COX-1, COX-2, mPGES-1, mPGES-2, and cPGES from $5 \mathrm{~h}$ to 3 days after collagenase-induced $\mathrm{ICH}$ in mice.

\section{Materials and methods}

\section{Animals}

This study was conducted in accordance with the National Institutes of Health guidelines for the use of experimental animals. Experimental protocols were approved by the Johns Hopkins University Animal Care and Use Committee. A total of 56 C57BL/6 male mice (25-35 g) were obtained from Charles River Laboratories (Wilmington, MA). All efforts were made to minimize the numbers of animals used and ensure minimal suffering.

\section{ICH model}

The procedure for modeling ICH by intrastriatal injection of collagenase was adapted to mice from an established rat protocol [20] and has been described previously $[21,22]$. Briefly, C57BL/6 mice weighing 22-35 g were anaesthetized by isoflurane $(3.0 \%$ for induction, $1.0 \%$ for maintenance) and ventilated with oxygen-enriched air (20\%:80\%) via a nose cone. We then injected mice in the left striatum with collagenase VII-S (0.075 U in $500 \mathrm{~nL}$ saline, C2399, Sigma, St. Louis, MO) at the following stereotactic coordinates: $1.0 \mathrm{~mm}$ anterior and $2.2 \mathrm{~mm}$ lateral of the bregma, $2.7 \mathrm{~mm}$ in depth. Collagenase was delivered over $5 \mathrm{~min}$. The needle was held in place for an additional 5 min to prevent reflux. Sham-operated mice were injected with saline only. Rectal temperature was monitored and maintained at $37.0 \pm$ $0.5^{\circ} \mathrm{C}$ with a heating pad throughout the experimental and recovery periods. This procedure resulted in reproducible lesions that were mostly restricted to the striatum.

\section{Preparation of brain slices}

At 5,24 , or $72 \mathrm{~h}$ after $\mathrm{ICH}, 3$ mice per group were anesthetized deeply with phenobarbital and perfused transcardially with phosphate-buffered saline (PBS), $\mathrm{pH}$ 7.4. Sham-operated control mice $(n=2$ at each time point) were perfused similarly. The brains were harvested and fixed with $4 \%$ paraformaldehyde overnight, cryoprotected in serial phosphate-buffered sucrose solutions $(20 \%, 30 \%$, and $40 \%)$ at $4{ }^{\circ} \mathrm{C}$, and then cut into $20-\mu \mathrm{m}$ sections with a cryostat.

\section{Hemorrhagic injury volume}

Mice $(n=5)$ were euthanized at $24 \mathrm{~h}$ after $\mathrm{ICH}$. The entire brain of each mouse was cut into $50-\mu \mathrm{m}$ sections with a cryostat. Sections were stained with Luxol fast blue (for myelin) and Cresyl Violet (for neurons) before being quantified for grey and white matter injury with SigmaScan Pro software (version 5.0.0 for Windows; Systat, San Jose, CA). The injury volume in cubic millimeters was calculated by multiplying the thickness by the damaged areas of each section [21].

\section{Immunofluorescence}

Immunofluorescence was carried out as described previously $[23,24]$. Briefly, free-floating sections were washed in PBS for 20 min, blocked in 5\% normal goat serum, and incubated overnight at $4{ }^{\circ} \mathrm{C}$ with primary antibodies: rabbit anti-COX-1 (1:250; Catalog No. 160109, Cayman Chemical, Ann Arbor, MI); rabbit antiCOX-2 (1:250; Catalog No. 160106, Cayman Chemical); rabbit anti-mPGES-1 (1:250; Catalog No. 160140, Cayman Chemical); rabbit anti-mPGES-2 (1:250; Catalog No. 160145, Cayman Chemical); rabbit anti-cPGES (1:250; Catalog No. 10209, Cayman Chemical); mouse anti-NeuN (1:500; Catalog No. MAB377, Chemicon, Temecula, CA), specific for neurons; rat anti-GFAP (1:250; Catalog No. 13-0300, Invitrogen, Carlsbad, CA), specific for astrocytes; and rat anti-CD11b (1:1000; Cata$\log$ No. MCA711G, Serotec, Raleigh, NC), specific for microglia/macrophages. The sections were then washed with PBS and incubated with Alexa-488 (1:1000; Molecular Probes, Eugene, OR)- and/or Cy3 (1:1000; Jackson Labs, West Grove, PA)-conjugated secondary antibodies for $60 \mathrm{~min}$. Stained sections $(\mathrm{n}=3 /$ mouse $)$ were examined with a fluorescence microscope; the images were captured from the frontoparietal cortex and striatum and analyzed by SPOT advanced image software (Diagnostic Instruments Inc., Sterling Heights, MI). Control sections were processed identically, except that primary antibodies were omitted. Control sections lacked specific 
staining. The specificity of the antibodies against COX1, COX-2, mPGES-1, mPGES-2, and cPGES was further confirmed by using the corresponding blocking peptides (Cayman Chemical).

To quantify the number of immunoreactive cells labeled with COX-1, COX-2, mPGES-1, mPGES-2, and cPGES, three sections per mouse (from the injection site and $360 \mu \mathrm{m}$ on each side; $\mathrm{n}=3$ mice/group) were analyzed in the perihematoma region of the striatum. This region was defined within one $20 \times$ field that corresponded to $\sim 460 \mu \mathrm{m}$ from the edge of the hematoma. Positively stained cells were counted in three comparable, randomly selected $30 \times$ microscopic fields. The numbers of immunoreactive cells from nine locations per mouse ( 3 fields per section $\times 3$ sections per mouse) were averaged and expressed as positive cells per field $(30 \times)$.

\section{Statistics}

All data are expressed as means \pm SD. The statistical comparisons among multiple groups were made using one-way ANOVA followed by Bonferroni correction. Statistical significance was set at $P<0.05$.

\section{Results}

\section{Brain injury volume}

Collagenase injection produced a hematoma that was primarily restricted to the striatum. All mice displayed obvious neurobehavioral deficits within hours of $\mathrm{ICH}$ onset (i.e., ipsilateral turning bias). At $24 \mathrm{~h}$ after $\mathrm{ICH}$, the brain injury volume calculated from serial sections stained with Luxol fast blue/Cresyl Violet via an image analysis system was $7.5 \pm 1.6 \mathrm{~mm}^{3}(\mathrm{n}=5)$.

\section{Expression of COX-1 and COX-2 after ICH}

COX-1 immunoreactivity was primarily observed in neuron-like and glia-like cells in the contralateral and ipsilateral striatum (figure 1A) and did not change significantly from $5 \mathrm{~h}$ to 3 days after ICH (figure 1A-D). To determine the cellular location of COX-1 following $\mathrm{ICH}$, we double stained serial sections obtained 3 days after $\mathrm{ICH}$ for $\mathrm{COX}-1$ and cell-specific antigens. The results revealed that $\mathrm{COX}-1$ immunoreactivity was mostly associated with neurons (figure 1B) and microglia (figure 1C). Co-localization was not observed with GFAP, a marker for astrocytes (figure 1D). Cell count analysis confirmed that the number of COX-1-immunoreactive neurons and microglia did not change from $5 \mathrm{~h}$ to 3 days in the perihematomal region of the striatum (figure $1 \mathrm{E}, \mathrm{n}=3$ /group, all $P>0.05$ ).

Immunoreactivity for COX-2 in neuron-like and glialike cells was minimal in the contralateral striatum (figure 2). After $\mathrm{ICH}$, the number of COX-2-immunoreactive cells increased in the perihematomal region of
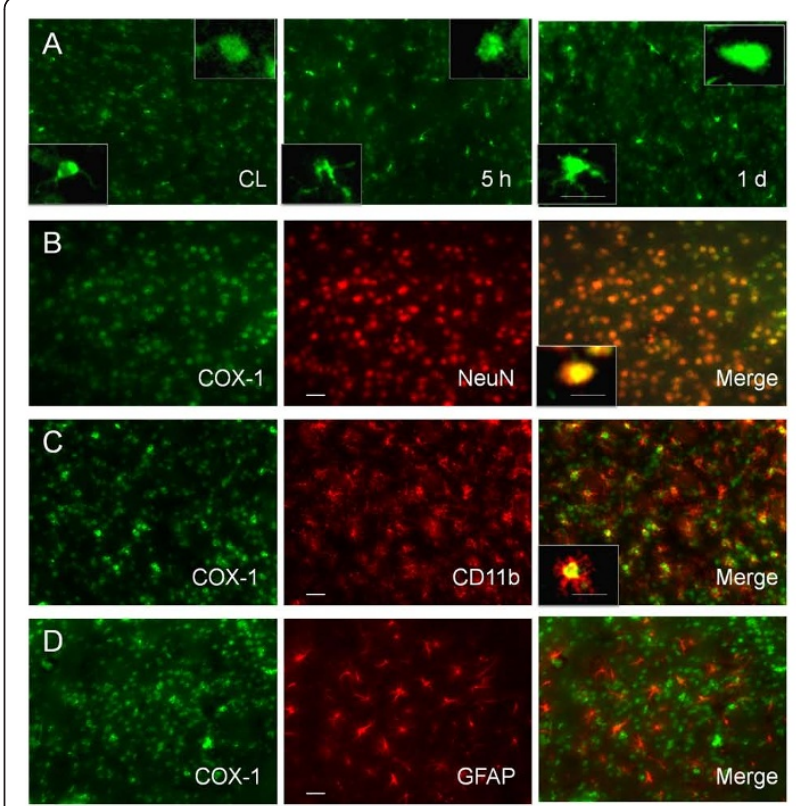

E

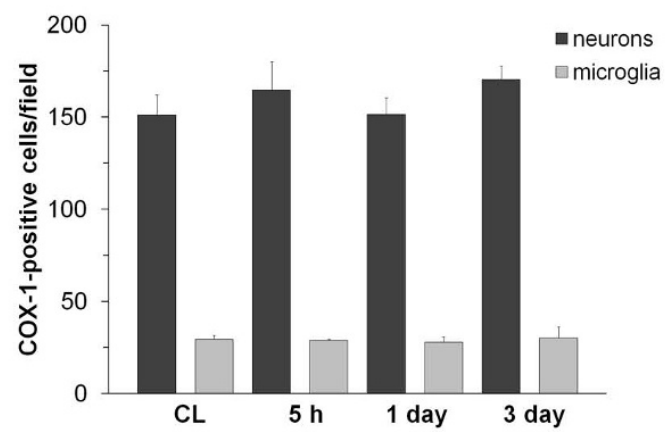

Figure 1 Immunostaining of COX-1 and cell type-specific proteins in the mouse brain after ICH. A: COX-1 was constitutively expressed in the contralateral striatum $(\mathrm{CL})$ and did not change significantly from $5 \mathrm{~h}$ to 1 day after $\mathrm{ICH}$. The insets in $\mathrm{A}$ are representative COX-1-expressing cells at higher magnification (Scale bar, $10 \mu \mathrm{m}$ ). B-D: Double immunostaining of COX-1 with cell type-specific markers for neurons (NeuN), microglia (CD11b), and astrocytes (GFAP) in the striatum surrounding the hematoma at 3 days after $\mathrm{ICH}$. Insets in B and $\mathrm{C}$ show a double-stained cell at higher magnification (Scale bar, 10 $\mu \mathrm{m})$. Scale bar in the middle panels of $B, C, D, 30 \mu \mathrm{m}$. E: Quantification analysis confirmed that the number of COX-1immunoreactive neurons and microglia did not change in the perihematomal region of the striatum from $5 \mathrm{~h}$ to 3 days after $\mathrm{ICH}$ ( $n=3$ /group, all $P>0.05)$. Values are means \pm S.D.

the striatum; the increase was present at $5 \mathrm{~h}$ and continued at 3 days, a finding confirmed by quantification analysis. COX-2-immunoreactive astrocytes displayed increasing hypertrophy of the cell bodies and processes from $5 \mathrm{~h}$ to 3 days after $\mathrm{ICH}$ (insets in figure 2). In the perihematomal region of the striatum, the immunoreactivity of COX-2 was significantly increased in neurons and astrocytes surrounding blood vessels at $5 \mathrm{~h}$. The 

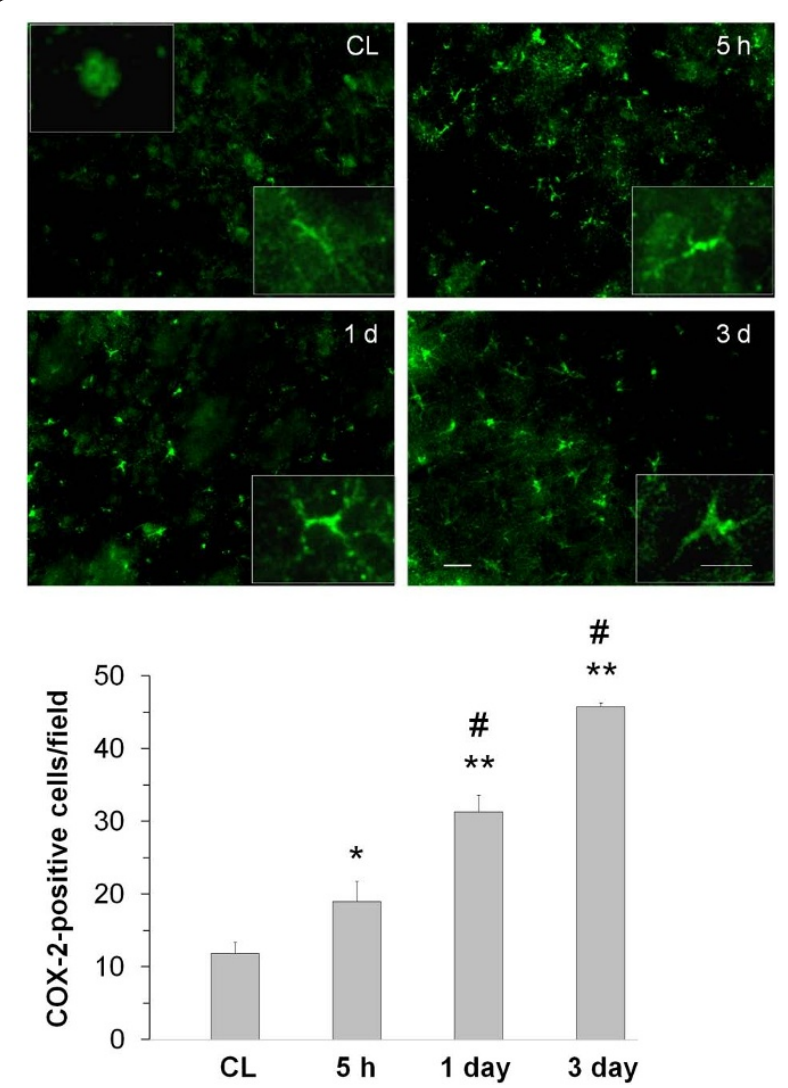

Figure 2 Immunostaining of COX-2 in the mouse brain after ICH. Minimal COX-2 immunoreactivity was observed in neuron-like and glia-like cells in the contralateral striatum $(\mathrm{CL})$. After $\mathrm{ICH}$, the number of strongly COX-2-immunoreactive cells increased in the perihematomal region of the striatum; the increase started at $5 \mathrm{~h}$ and continued at 3 days. Scale bar, $30 \mu \mathrm{m}$. Insets are representative COX-2-expressing cells at higher magnification (Scale bar, $15 \mu \mathrm{m}$ ). Quantification analysis confirmed that the number of strongly COX2-immunoreactive cells increased from $5 \mathrm{~h}$ to 3 days in the perihematomal region of the striatum $\left(n=3 /\right.$ group, ${ }^{*} P<0.05$, ** $P<0.01$ compared with the $\mathrm{CL}_{1}{ }^{\#} P<0.01$ compared with the previous time point). Values are means \pm S.D.

intensity tended to decrease in neurons and increase in astrocytes at 1 day after $\mathrm{ICH}$. At 3 days, the immunoreactivity of COX-2 continued to increase in astrocytes but was nearly undetectable in neurons (figure 3 ). COX2 immunoreactivity was not detected in microglia at any time point studied (figure 3 ).

\section{Expression of mPGES-1, mPGES-2, and CPGES after ICH}

Minimal immunoreactivity of mPGES-1 was observed in neuron-like cells in the contralateral cortex (data not shown) and striatum (figure 4A). In the perihematomal region, the immunoreactivity of mPGES-1 began to increase in glia-like cells at 1 day (figure 4C) and was further increased at 3 days after ICH (figure 4D). In the ipsilateral cortex, mPGES-1 immunoreactivity was
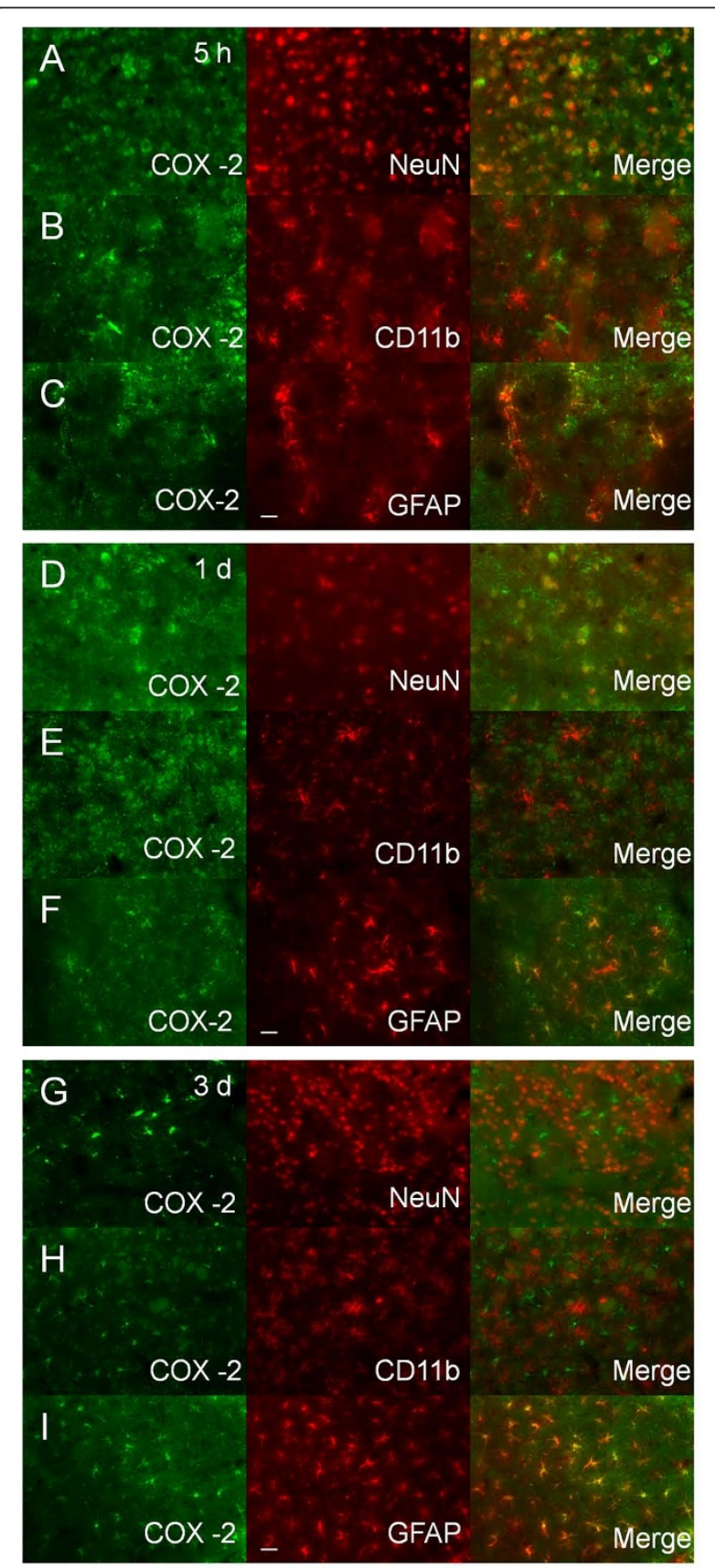

Figure 3 Double immunostaining of COX-2 with cell typespecific markers (NeuN for neurons, CD11b for microglia, and GFAP for astrocytes) in the mouse brain after ICH. A-C: In the perihematomal region of the striatum, the number of COX-2immunoreactive cells increased; double immunostaining demonstrated that COX-2 was expressed in neurons (A) and astrocytes (C), but not in microglia (B), at $5 \mathrm{~h}$ after ICH. D-F: At 1 day after $\mathrm{ICH}$, the COX-2 immunoreactivity tended to decrease in neurons (D) and increase in astrocytes (F) of the perihematomal region in the striatum; however COX-2 immunoreactivity remained unchanged in microglia (E). G-I: At 3 days post- $\mathrm{ICH}, \mathrm{COX}-2$ immunoreactivity further increased in astrocytes (I) but was not observed in neurons $(\mathrm{G})$ or microglia $(\mathrm{H})$ in the perihematomal region of the striatum. Scale bar in C, F, I, $30 \mu \mathrm{m}$. 

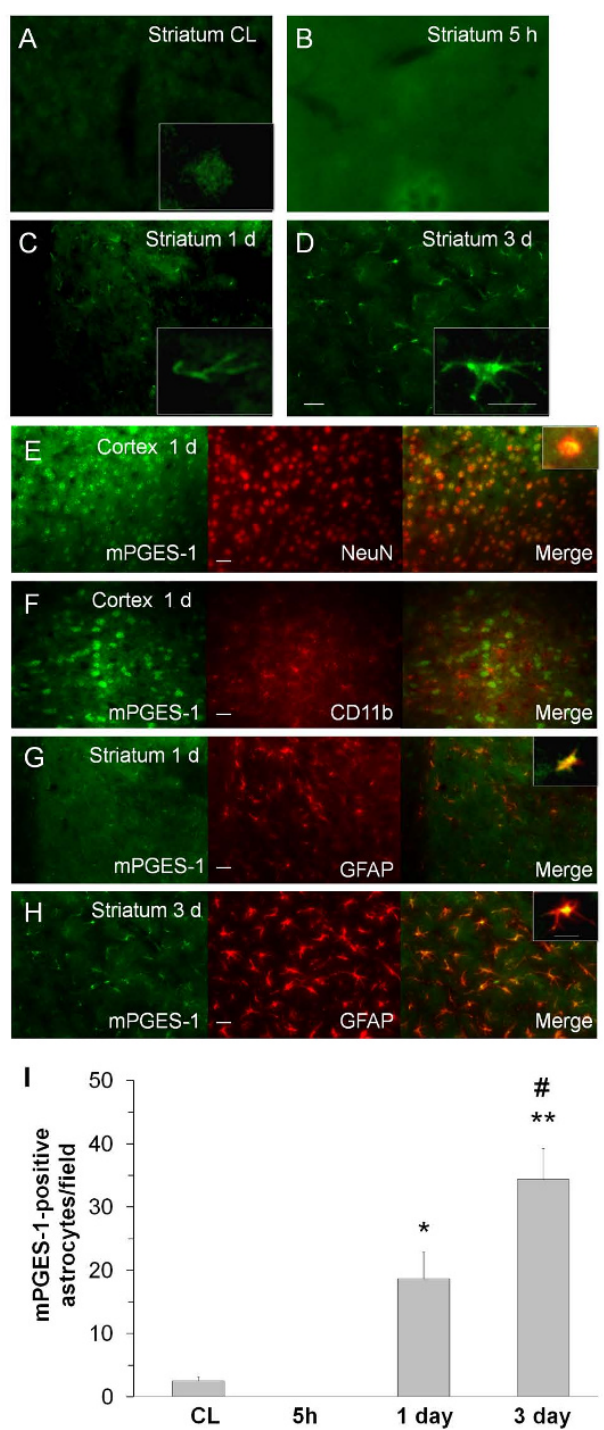

Figure 4 Immunostaining of mPGES-1 and cell type-specific proteins (NeuN for neurons, CD11b for microglia, and GFAP for astrocytes) in the mouse brain after ICH. (A) Minimal immunoreactivity of mPGES-1 was detected in neuron-like cells in the contralateral striatum $(\mathrm{CL})$. B-D: In the perihematomal region of the striatum, mPGES-1 immunoreactivity was undetectable at $5 \mathrm{~h}$ (B) but began to increase in glia-like cells at 1 day (C). It was additionally increased in glia-like cells at 3 days (D). Insets in A, C, and $D$ are representative $\mathrm{MPGES}$-1-expressing cells at higher magnification (Scale bar, $15 \mu \mathrm{m}$ ). E-F: Double immunostaining showed induction of mPGES-1 in neurons (E), but not in microglia $(F)$, in the ipsilateral frontal-parietal cortex at 1 day. $\mathrm{G}-\mathrm{H}$ : In the perihematomal region of the striatum, double immunostaining showed that MPGES-1 was induced primarily in astrocytes at 1 day (G); the number of mPGES-1-immunoreactive astrocytes was additionally increased at 3 days $(H)$. Scale bar in D-H, $30 \mu \mathrm{m}$. Insets in $E, G$, and $H$ are double-stained cells at higher magnification (Scale bar, $15 \mu \mathrm{m}$ ). Quantification analysis (I) confirmed that the number of mPGES-1 immunoreactive cells increased at 1 day, and further increased at 3 days in the perihematomal region of the striatum ( $n=3$ /group, ${ }^{*} P<0.05$, ** $P<0.01$ compared with the $C L$, $\# P<0.01$ compared with day 1). Values are means \pm S.D. observed primarily in neurons (figure 4E), infrequently in astrocytes (data not shown), and rarely in microglia/ macrophages (figure 4F) at 1 day after ICH. In the ipsilateral striatum, however, mPGES-1 immunoreactivity was detected primarily in astrocytes; it was elevated at 1 day (figure 4G) and further increased at 3 days (figure $4 \mathrm{H}$ ), as confirmed by quantification analysis (Figure 4I). mPGES-1 was not detected in neurons (data not shown).

mPGES-2 was constitutively expressed in neuron-like cells in the contralateral and ipsilateral striatum (figure 5A-D) and did not change significantly between $5 \mathrm{~h}$ and 3 days after ICH (figure 5B-D). Double immunofluorescence labeling revealed that the mPGES-2 was located primarily in neurons at $5 \mathrm{~h}$ after ICH (figure $5 \mathrm{E}$ ) but that it was absent from microglia/macrophages at 1 day (figure 5F) and astrocytes at 3 days (figure 5G) after $\mathrm{ICH}$. Cell count analysis confirmed that the number of mPGES-2-immunoreactive cells did not change from 5 $\mathrm{h}$ to 3 days in the perihematomal region of the striatum (figure $5 \mathrm{H}, \mathrm{n}=3$ /group, all $P>0.05$ ).

Similar to mPGES-2, immunostaining revealed cPGES to be expressed constitutively in neuron-like cells in the contralateral and ipsilateral striatum (figure 6A-C); it did not change significantly between 1 and 3 days after $\mathrm{ICH}$ (figure 6B-C). Double immunofluorescence labeling indicated that cPGES was present primarily in neurons (figure 6D), but not in microglia/macrophages (figure 6E) or astrocytes (figure 6F) at 1 day after ICH. Cell count analysis confirmed that the number of cPGESimmunoreactive cells did not change from 1 day to 3 days in the perihematomal region of the striatum (figure 6G, n $=3$ /group, all $P>0.05$ ).

\section{Discussion}

To our knowledge, this is the first systematic study performed to characterize the expression and cellular localization of COX and PGES isozymes in the hemorrhagic brain of mice. Using immunofluorescence staining, we observed constitutive expression of COX-1, mPGES-2, and cPGES in neurons; COX-1 was also constitutively expressed in microglia. In contrast, COX-2 and mPGES1 immunoreactivity, which was minimal in the normal brain, underwent distinct time-dependent changes in neurons and astrocytes of the perihematomal region during the first 3 days post-ICH. Our data support the premise that the COX/PGES signaling pathway contributes to ICH pathology.

COX-1 is constitutively expressed in most tissues [9]. We demonstrated for the first time that COX-1 is constitutively expressed in neurons and microglia in the hemorrhagic brain. Although involvement of COX-1 in $\mathrm{ICH}$ pathology has not been studied, the evidence that COX-1 is produced in microglia of the perihematomal 

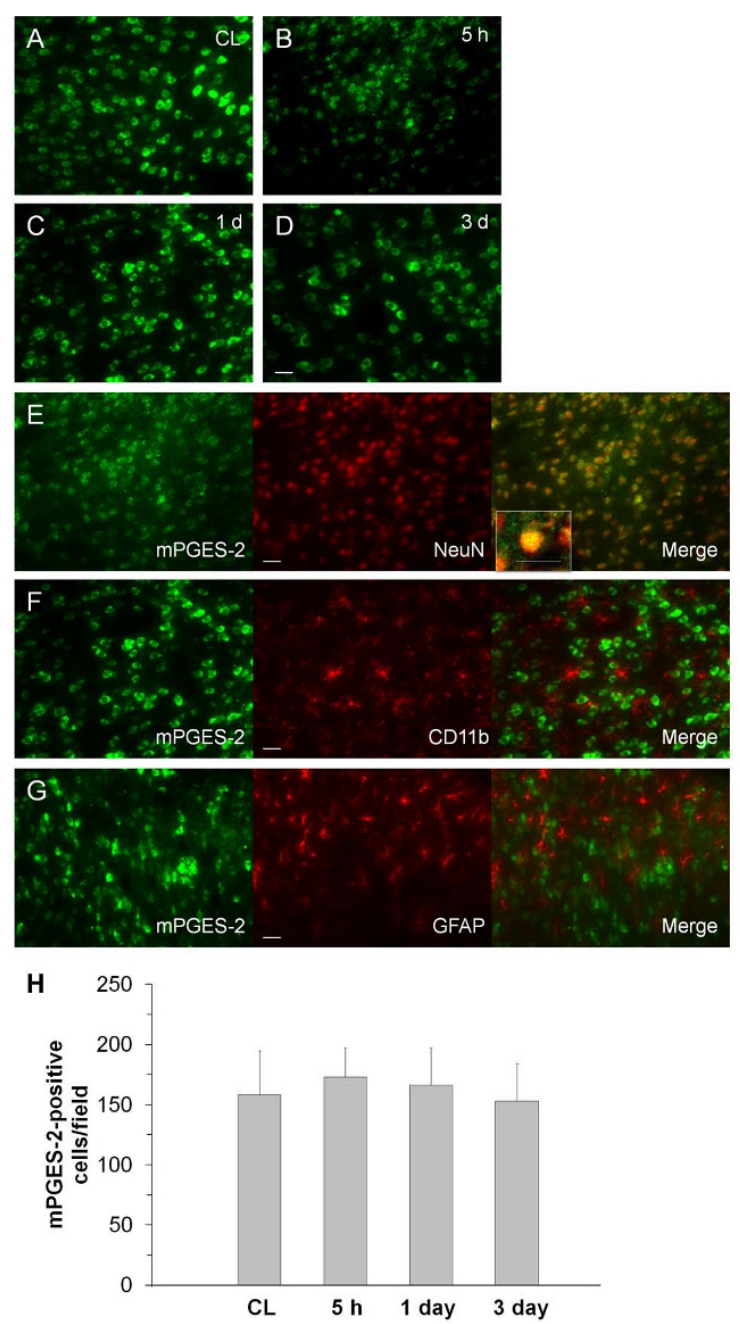

Figure 5 Immunostaining of mPGES-2 and cell type-specific proteins (NeuN for neurons, CD11b for microglia, and GFAP for astrocytes) in the mouse brain after ICH. A: Immunoreactive staining for mPGES-2 was observed in neuron-like cells in the contralateral striatum (CL). B-D: In the perihematomal region of the striatum, mPGES-2 immunoreactivity did not change significantly between $5 \mathrm{~h}$ (B) and 1 day (C) or 3 days (D) post-ICH. E-G: In the perihematomal region of the striatum, double immunostaining revealed that mPGES-2 was present in neurons at 1 day $(\mathrm{E})$, but not in microglia at 1 day (F) or astrocytes at 3 days (G). Scale bar in D-G, $30 \mu \mathrm{m}$. Inset in $\mathrm{E}$ is a double-stained cell at higher magnification (Scale bar, $20 \mu \mathrm{m}$ ). Quantification analysis $(\mathrm{H})$ confirmed that the number of mPGES-2-immunoreactive cells did not change in the perihematomal region of the striatum from $5 \mathrm{~h}$ to 3 days after $\mathrm{ICH}$ ( $n=3$ /group, all $P>0.05)$. Values are means \pm S.D.

region implies a toxic role of COX-1 in the pathophysiology of the disease. This hypothesis is based on the fact that activated microglia/macrophages are the major sources of proinflammatory mediators $[1,3]$ and that inhibition of microglial activation before or early after ICH decreases neuronal death and improves neurologic function $[21,25]$. We therefore propose that microglial
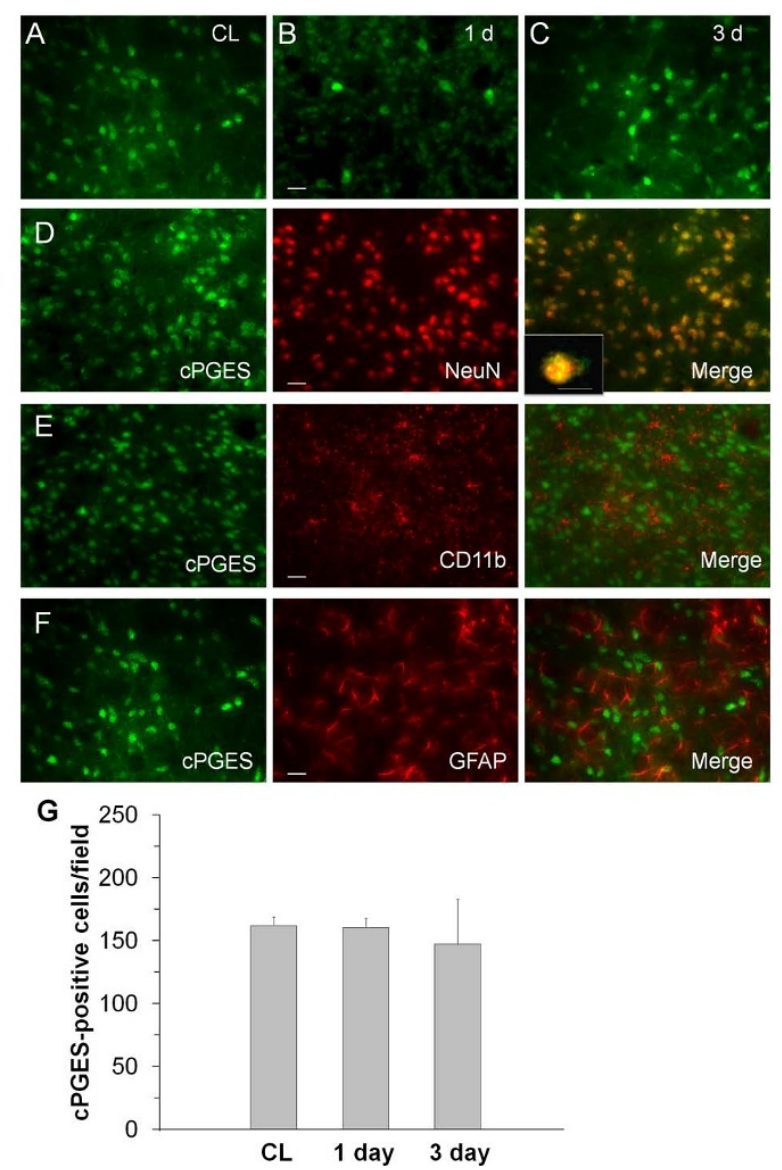

Figure 6 Immunostaining of CPGES and cell type-specific proteins (NeuN for neurons, CD11b for microglia, and GFAP for astrocytes) in the mouse brain after ICH. A: Immunoreactive staining for CPGES was observed in neuron-like cells in the contralateral striatum (CL). B-C: In the perihematomal region of the striatum, CPGES immunoreactivity did not change significantly between 1 day (B) and 3 days (C). D-F: In the perihematomal region of the striatum, double immunostaining showed that CPGES was present in neurons (D), but not in microglia (E) or astrocytes (F), at 1 day. Scale bar in B-F, $30 \mu \mathrm{m}$. Inset in $\mathrm{D}$ is a double-stained cell at higher magnification (Scale bar, $15 \mu \mathrm{m}$ ). Quantification analysis (G) confirmed that the number of CPGES immunoreactive cells did not change in the perihematomal region of the striatum between 1 day and 3 days after $\mathrm{ICH}(\mathrm{n}=3 /$ group, all $P>0.05)$. Values are means \pm S.D.

COX-1 might immediately initiate synthesis of prostaglandins in response to microglial activation and could be considered one of the major players in mediating neuroinflammation after $\mathrm{ICH}$.

In the normal brain, COX-2 is constitutively expressed in neurons of the cortex, hippocampus, and striatum [8]. COX-2 is mainly induced in response to inflammatory stimuli; deletion of the COX-2 gene or selective COX-2 inhibition reduces infarction volume and neuronal death after cerebral ischemia [26-28]. To our knowledge, only two studies have investigated post-ICH COX-2 
expression, and the results were conflicting $[18,19]$. Zhao et al. [19] demonstrated that COX-2 mRNA and protein were increased within $3 \mathrm{~h}$ after $\mathrm{ICH}$ and that COX-2 immunoreactivity was increased in blood vessels and neurons in the perihematomal region at $4 \mathrm{~h}$; the increase in immunoreactivity was transient, followed by a significant down-regulation at days 1 and 3 . In the present study, we found that the immunoreactivity of COX-2 in astrocytes increased gradually in the perihematomal region from $5 \mathrm{~h}$ to 3 days, whereas neuronal COX-2 increased only transiently at $5 \mathrm{~h}$ after $\mathrm{ICH}$. Most of our results are consistent with the findings by Gong et al. [18], except that we found COX-2 to be increasingly induced in astrocytes from 1 to 3 days, whereas Gong et al. reported that COX-2 was induced in endothelial cells, perivascular cells, and infiltrating leukocytes at 1 day after blood infusion. The reason for the discrepancy between the previous studies and our own is not clear, but it may be a result of differences in $\mathrm{ICH}$ models and species used and the size of the intrastriatal hematoma formed. In the two previous studies, investigators modeled $\mathrm{ICH}$ by injecting rats intrastriatally with differing amounts of autologous blood. In contrast, we used a collagenase-induced ICH model in mice that may cause gradual hematoma growth over the first few hours with subsequent inflammation. Our data suggest that astrocytic COX-2 in concert with microglial COX-1 might contribute to collagenase-induced post-hemorrhagic neuroinflammation. More studies are warranted to understand whether the functions of neuronal and astrocytic COX-2 differ after ICH.

Induction of mPGES-1 expression has been observed in various conditions, such as inflammation, fever, pain, tissue repair, and cancer, in which COX-2-derived $\mathrm{PGE}_{2}$ plays a critical role [29]. In the ischemic brain, mPGES1 and COX-2 are both induced in neurons, microglia, and endothelial cells in the ipsilateral cerebral cortex and striatum [7]. It has been confirmed that mPGES-1 and COX-2 are co-localized and co-induced in the infarct region of the cortex, and it has been suggested that they act together to exacerbate stroke injury [14]. At 1 day post-ICH in our model, mPGES-1 expression in the ipsilateral cortex was elevated primarily in neurons whereas in the perihematomal region, it was elevated in astrocytes. Astrocytic expression continued to increase for at least 3 days. However, we observed no apparent changes in the expression of neuronal mPGES2 or cPGES. The different cellular expression profiles for mPGES-1 between cortex and striatum suggests that mPGES-1 may be involved in different signaling pathways within the cortical neurons and striatal astrocytes after ICH. In line with the results from a lipopolysaccharide-induced inflammation model [30], we found that the induction of $\mathrm{COX}-2$ protein expression was more rapid than that of mPGES-1 in the hemorrhagic brain. These results suggest that the sequential up-regulation and co-induction of COX-2 and mPGES-1 in astrocytes in the perihematomal region might contribute to inflammation-mediated secondary brain injury after $\mathrm{ICH}$, possibly through excessive $\mathrm{PGE}_{2}$ production.

In conclusion, our data provide novel evidence that COX-1, mPGES-2, and cPGES are constitutively expressed in the hemorrhagic brain; COX-2 is induced early in neurons and later in astrocytes. Although neuronal COX-2 is induced earlier than astrocytic COX-2, the latter is induced in parallel with astrocytic mPGES1. Together, our data suggest that microglial COX-1, neuronal COX-2, astrocytic COX-2, and astrocytic mPGES-1 may work sequentially to affect ICH outcomes. Based on our previous observations that neuroinflammation affects the normal function of the entire brain in patients with lethal $\mathrm{ICH}$ [31], these findings have implications for efforts to develop anti-inflammatory strategies that target the COX/PGES pathway to reduce $\mathrm{ICH}$-induced secondary brain damage. Indeed, a recent study showed that inhibition of COX-2 attenuated inflammation, neuronal death, and gliosis and promoted long-term recovery in motor function and myelination in rabbit pups with intraventricular hemorrhage [32].

\section{Acknowledgements}

This work was supported by AHA 09BGIA2080137 and NIH K01AG031926 (JW). Dr. Tao Wu was partially supported by a training grant from Changhai Hospital, Second Military Medical University, PR China. We thank Claire Levine for assistance with manuscript preparation.

\section{Author details}

'Department of Anesthesiology/Critical Care Medicine, Johns Hopkins University, School of Medicine, Baltimore, MD, USA. ${ }^{2}$ Department of Neurology, Changhai Hospital, Second Military Medical University, Shanghai 200433, PR China. ${ }^{3}$ Department of Pathology, First Clinical Hospital, Harbin Medical University, Harbin 150001, PR China.

\section{Authors' contributions}

TW, HW, and Jessica W carried out the ICH model and immunofluorescence staining and helped draft the manuscript. Jian W conceived of the study, participated in its design and conduct, and helped draft the manuscript. All authors read and approved the final manuscript.

\section{Competing interests}

The authors declare that they have no competing interests.

Received: 4 December 2010 Accepted: 8 March 2011

Published: 8 March 2011

\section{References}

1. Wang J: Preclinical and clinical research on inflammation after intracerebral hemorrhage. Prog Neurobiol 2010, 92:463-477.

2. Qureshi Al, Mendelow AD, Hanley DF: Intracerebral haemorrhage. Lancet 2009, 373:1632-1644.

3. Wang J, Doré S: Inflammation after intracerebral hemorrhage. J Cereb Blood Flow Metab 2007, 27:894-908.

4. Xue $\mathrm{M}$, Yong $\mathrm{W}$ : Matrix metalloproteinases in intracerebral hemorrhage. Neurol Res 2008, 30:775-782. 
5. Xi G, Keep RF, Hoff JT: Mechanisms of brain injury after intracerebral haemorrhage. Lancet Neurol 2006, 5:53-63.

6. Iadecola C: Cyclooxygenase-2 and stroke: the long and short of it. Ann Neurol 2003, 54:141-142.

7. Ikeda-Matsuo Y, Ota A, Fukada T, Uematsu S, Akira S, Sasaki Y: Microsomal prostaglandin E synthase-1 is a critical factor of stroke-reperfusion injury. Proc Natl Acad Sci USA 2006, 103:11790-11795.

8. Hewett SJ, Bell SC, Hewett JA: Contributions of cyclooxygenase-2 to neuroplasticity and neuropathology of the central nervous system. Pharmacol Ther 2006, 112:335-357.

9. Phillis JW, Horrocks LA, Farooqui AA: Cyclooxygenases, lipoxygenases, and epoxygenases in CNS: their role and involvement in neurological disorders. Brain Res Rev 2006, 52:201-243.

10. Yang $H$, Chen C: Cyclooxygenase-2 in synaptic signaling. Curr Pharm Des 2008, 14:1443-1451.

11. Jakobsson PJ, Thoren S, Morgenstern R, Samuelsson B: Identification of human prostaglandin E synthase: a microsomal, glutathione-dependent, inducible enzyme, constituting a potential novel drug target. Proc Natl Acad Sci USA 1999, 96:7220-7225.

12. Murakami M, Nakatani $Y$, Tanioka T, Kudo I: Prostaglandin E synthase. Prostaglandins Other Lipid Mediat 2002, 68-69:383-399.

13. Ikeda-Matsuo Y, Tanji H, Ota A, Hirayama Y, Uematsu S, Akira S, Sasaki Y: Microsomal prostaglandin E synthase-1 contributes to ischaemic excitotoxicity through prostaglandin E2 EP3 receptors. $\mathrm{Br} J$ Pharmacol 2010, 160:847-859.

14. Ikeda-Matsuo Y, Hirayama Y, Ota A, Uematsu S, Akira S, Sasaki Y: Microsomal prostaglandin E synthase- 1 and cyclooxygenase- 2 are both required for ischaemic excitotoxicity. Br J Pharmacol 2010, 159:1174-1186.

15. Andreasson $\mathrm{K}$ : Prostaglandin signalling in cerebral ischaemia. $\mathrm{Br} \mathrm{J}$ Pharmacol 2010, 160:844-846.

16. Choi SH, Aid S, Bosetti F: The distinct roles of cyclooxygenase- 1 and -2 in neuroinflammation: implications for translational research. Trends Pharmacol Sci 2009, 30:174-181.

17. Andreasson K: Emerging roles of PGE2 receptors in models of neurological disease. Prostaglandins Other Lipid Mediat 2010, 91:104-112.

18. Gong C, Ennis SR, Hoff JT, Keep RF: Inducible cyclooxygenase-2 expression after experimental intracerebral hemorrhage. Brain Res 2001, 901:38-46.

19. Zhao X, Zhang Y, Strong R, Zhang J, Grotta JC, Aronowski J: Distinct patterns of intracerebral hemorrhage-induced alterations in NFkappaB subunit, iNOS, and COX-2 expression. J Neurochem 2007, 101:652-663.

20. Rosenberg GA, Mun-Bryce S, Wesley M, Kornfeld M: Collagenase-induced intracerebral hemorrhage in rats. Stroke 1990, 21:801-807.

21. Wang J, Rogove AD, Tsirka AE, Tsirka SE: Protective role of tuftsin fragment 1-3 in an animal model of intracerebral hemorrhage. Ann Neurol 2003, 54:655-664

22. Grossetete $M$, Rosenberg GA: Matrix metalloproteinase inhibition facilitates cell death in intracerebral hemorrhage in mouse. J Cereb Blood Flow Metab 2008, 28:752-763.

23. Wang J, Tsirka SE: Neuroprotection by inhibition of matrix metalloproteinases in a mouse model of intracerebral haemorrhage. Brain 2005, 128:1622-1633.

24. Wang J, Doré S: Heme oxygenase-1 exacerbates early brain injury after intracerebral haemorrhage. Brain 2007, 130:1643-1652.

25. Wang J, Tsirka SE: Tuftsin fragment 1-3 is beneficial when delivered after the induction of intracerebral hemorrhage. Stroke 2005, 36:613-618.

26. Candelario-Jalil E, Gonzalez-Falcon A, Garcia-Cabrera M, Alvarez D, AlDalain S, Martinez G, Leon OS, Springer JE: Assessment of the relative contribution of COX-1 and COX-2 isoforms to ischemia-induced oxidative damage and neurodegeneration following transient global cerebral ischemia. J Neurochem 2003, 86:545-555.

27. Sasaki T, Kitagawa K, Yamagata K, Takemiya T, Tanaka S, Omura-Matsuoka E, Sugiura S, Matsumoto M, Hori M: Amelioration of hippocampal neuronal damage after transient forebrain ischemia in cyclooxygenase-2-deficient mice. J Cereb Blood Flow Metab 2004, 24:107-113.

28. Candelario-Jalil E, Gonzalez-Falcon A, Garcia-Cabrera M, Leon OS, Fiebich BL: Post-ischaemic treatment with the cyclooxygenase-2 inhibitor nimesulide reduces blood-brain barrier disruption and leukocyte infiltration following transient focal cerebral ischaemia in rats. $J$ Neurochem 2007, 100:1108-1120.
29. Thoren S, Weinander R, Saha S, Jegerschold C, Pettersson PL, Samuelsson B, Hebert $H$, Hamberg $M$, Morgenstern $R$, Jakobsson PJ: Human microsomal prostaglandin E synthase-1: purification, functional characterization, and projection structure determination. J Biol Chem 2003, 278:22199-22209.

30. Ikeda-Matsuo $Y$, Ikegaya $Y$, Matsuki N, Uematsu S, Akira S, Sasaki Y: Microglia-specific expression of microsomal prostaglandin E2 synthase-1 contributes to lipopolysaccharide-induced prostaglandin E2 production. J Neurochem 2005, 94:1546-1558.

31. Wu H, Zhang Z, Hu X, Zhao R, Song Y, Ban X, Qi J, Wang J: Dynamic changes of inflammatory markers in brain after hemorrhagic stroke in humans: a postmortem study. Brain Res 2010, 1342:111-117.

32. Vinukonda G, Csiszar A, Hu F, Dummula K, Pandey NK, Zia MT, Ferreri NR, Ungvari Z, LaGamma EF, Ballabh P: Neuroprotection in a rabbit model of intraventricular haemorrhage by cyclooxygenase-2, prostanoid receptor1 or tumour necrosis factor-alpha inhibition. Brain 2010, 133:2264-2280.

doi:10.1186/1742-2094-8-22

Cite this article as: Wu et al:: Expression and cellular localization of cyclooxygenases and prostaglandin E synthases in the hemorrhagic brain. Journal of Neuroinflammation 2011 8:22

\section{Submit your next manuscript to BioMed Central and take full advantage of:}

- Convenient online submission

- Thorough peer review

- No space constraints or color figure charges

- Immediate publication on acceptance

- Inclusion in PubMed, CAS, Scopus and Google Scholar

- Research which is freely available for redistribution

Submit your manuscript at www.biomedcentral.com/submit
C) Biomed Central 